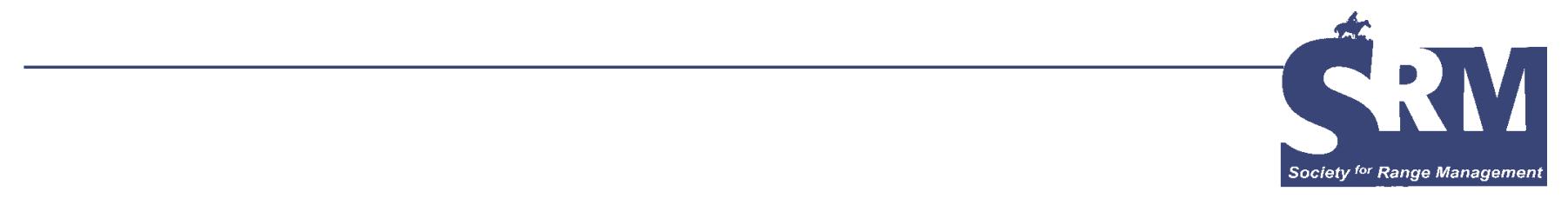

\title{
Managing Medusahead in the Intermountain West Is at a Critical Threshold
}

\section{By Kirk W. Davies and Dustin D. Johnson}

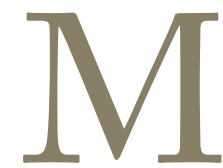

edusahead (Taeniatherum caput-medusae [L.] Nevski) invasion of Intermountain West rangelands is approaching a crossroads. Either an aggressive, hard-hitting effort to prevent its spread into new areas is initiated or millions of acres will be lost. Medusahead is an aggressive, exotic, annual grass invading rangelands in the western United States. ${ }^{1}$ While large expanses of rangeland in the Intermountain West have been invaded by medusahead or are threatened by its invasion,,$^{1,2}$ the amount of land actually infested by medusahead is still small relative to the area it could potentially invade in the future. By the early 1990s, 14 million acres of public lands in the Intermountain West were infested with medusahead, cheatgrass (Bromus tectorum L.), or both; however, the area at risk of invasion by these two grasses is at least 60 million acres. ${ }^{2}$ And unfortunately, medusahead is already spreading rapidly. For example, its extent increased from 18 to 31 of Oregon's 36 counties between 1962 and 2004. ${ }^{3,4}$ In Idaho, rangelands infested by medusahead more than doubled between 1957 and 1992.5

The rapid spread of medusahead is a serious management concern because it reduces grazing capacity by up to $80 \%$, degrades wildlife habitat, decreases biodiversity, and potentially alters ecosystem functions (Photo 1). ${ }^{1,6}$ An alarming but rarely mentioned impact of medusahead invasion is that it may exacerbate the decline of sagebrush-obligate wildlife species, like sage-grouse, as it replaces plant communities providing critical habitat. Medusahead replaces desirable vegetation directly by competition and suppression and indirectly by an increase in fire frequency. Medusahead can effectively out-compete desirable vegetation for soil nutrients and water. ${ }^{7-9}$ Medusahead litter has a slow decomposition rate, because of high silica content, allowing it to accumulate over time and suppress desirable plants. ${ }^{10}$ The

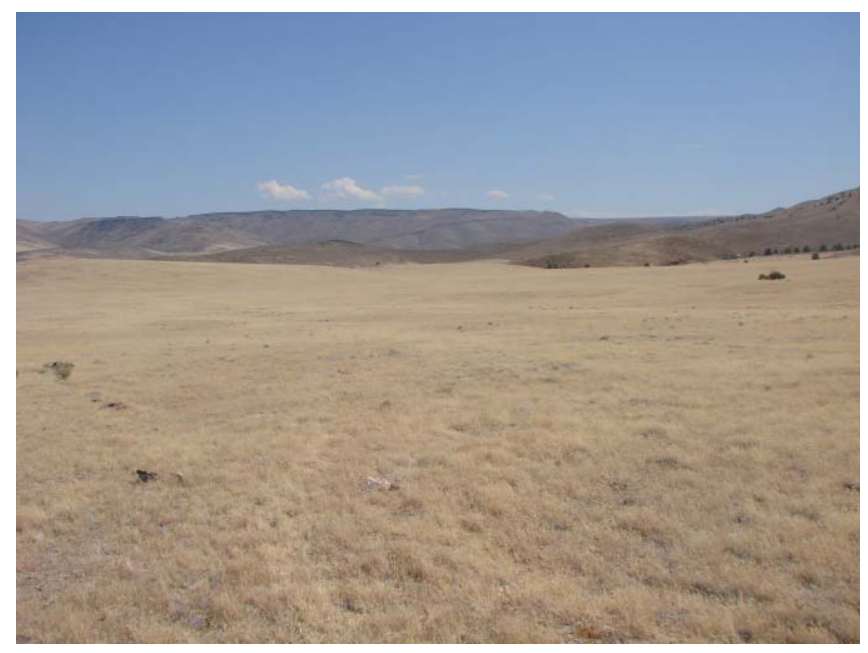

Photo 1. Sagebrush steppe communities that have been replaced with medusahead.

buildup of medusahead litter also increases the amount and continuity of fine fuel, which can increase fire frequency to the detriment of desirable vegetation.,11,12

Temporary control of near monocultures of medusahead can be achieved with herbicides, ${ }^{13,14}$ but revegetation is expensive and often unsuccessful in the Intermountain West (Photo 2). ${ }^{1,12,15}$ Revegetation success is low because climatic conditions rarely favor seedling establishment of desirable species and in the absence of competition, medusahead returns to dominate the site in a short period of time..$^{1,12}$

Because restoration is so difficult, resources would be better spent preventing medusahead from spreading, and controlling new infestations with enough desirable vegetation remaining that revegetation would not be necessary. 


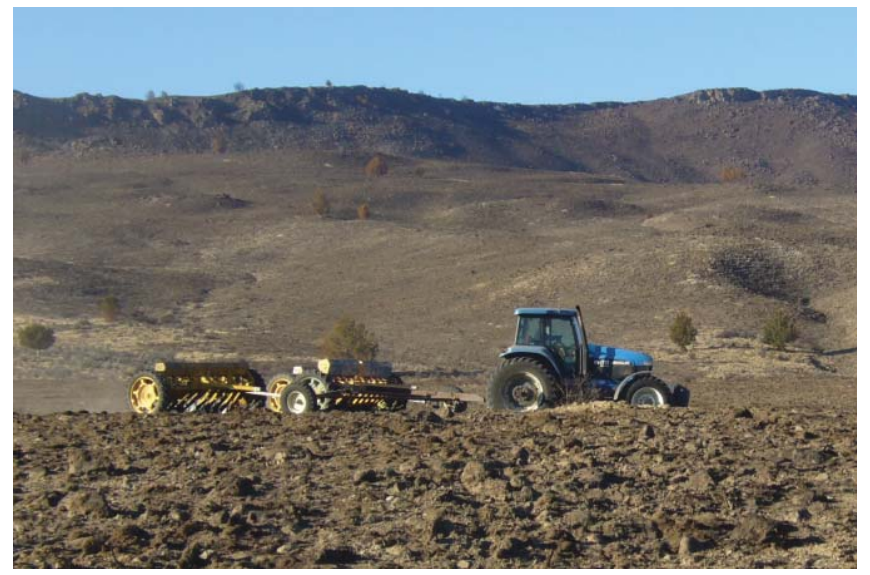

Photo 2. Restoration effort after a wildfire in medusahead-invaded plant communities.

Preventing weed invasions has been demonstrated to be more cost-effective than revegetation attempts after weed invasion. ${ }^{16-18}$ Prevention of further medusahead invasions should focus on three strategies: 1) preventing medusahead seeds from dispersing to new locations, 2) increasing the resistance of desirable plant communities to medusahead invasion, and 3) searching for and eradicating new infestations.

Medusahead seeds have long barbed awns that facilitate their dispersal by vehicles and animals (Photo 3)..$^{14,19}$ Decreasing animal and vehicle contact with medusahead when its seeds can be dispersed would limit its spread. ${ }^{20}$ Containment zones (areas where a weed is not allowed to complete its life cycle) around medusahead infestations require a width of only a few meters to be effective because, in the absence of human, livestock, and vehicle contact, medusahead seeds disperse relatively short distances. ${ }^{20}$

If medusahead seeds are dispersing into non-invaded areas, the resistance of the plant community to medusahead establishment is critical. Plant communities that are resistant to medusahead invasion have higher densities of large perennial bunchgrasses than communities that are less resistant; ${ }^{20}$ thus, managing rangelands to promote and maintain large perennial bunchgrasses is critical for plant community resistance to medusahead invasion. Preventing the spread of medusahead and increasing desirable plant community resistance to invasion will reduce the establishment of new infestations; however, successful medusahead management will also require searching for and eradicating new infestations. Controlling new weed infestations is a more effective strategy than trying to control large infestations. ${ }^{21,22}$

Even if endeavors to prevent and control new medusahead infestations are not completely successful, these efforts will slow the rate of spread and afford researchers and land managers more time to develop better prevention, restoration, and control methods. Furthermore, slowing the rate of invasion helps promote rangeland health and productivity on areas at risk of medusahead invasion.

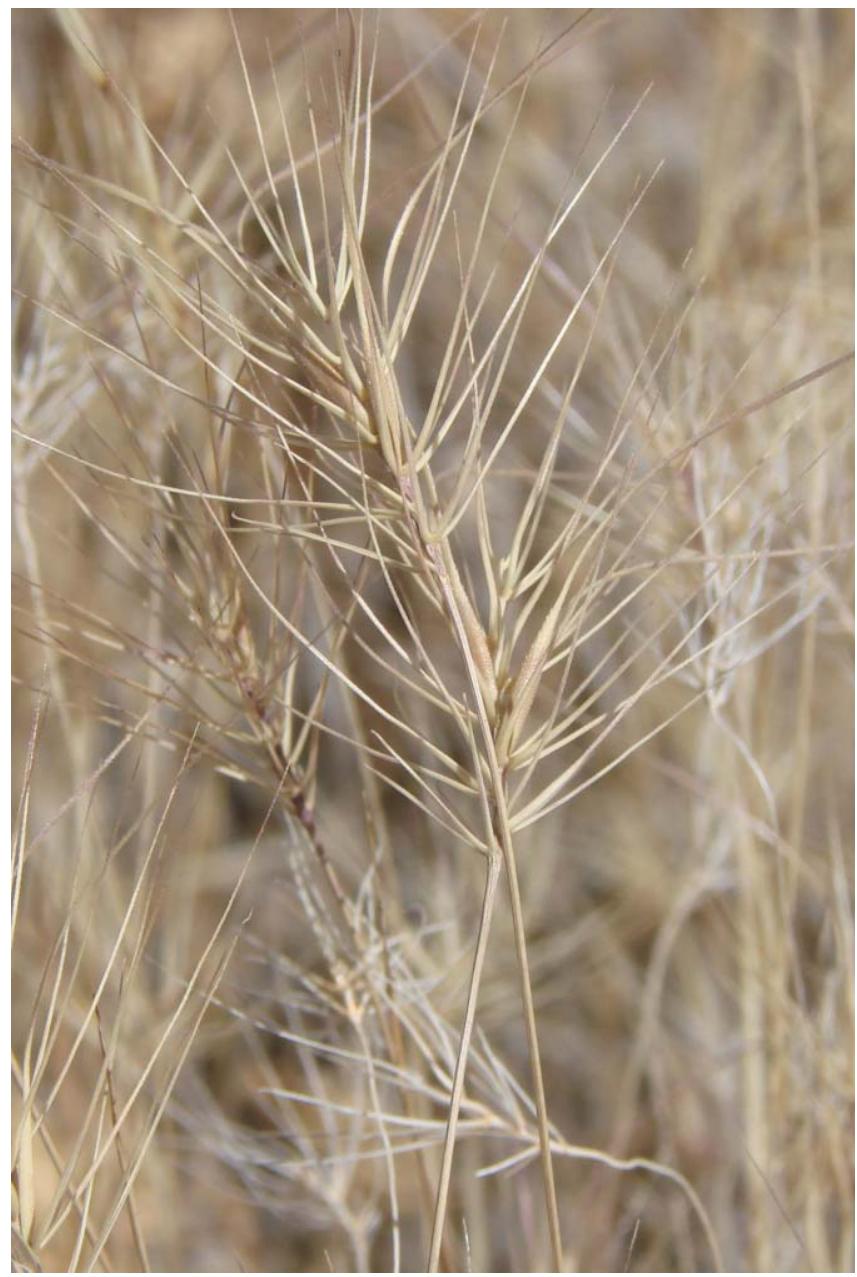

Photo 3. Close-up of medusahead seed heads. Notice the long awns on the medusahead seeds.

Medusahead is a very serious threat to rangelands of the Intermountain West. Without an active prevention program, medusahead will continue to expand, increasing its negative economic and ecological impacts. A well organized and adequately funded effort to prevent medusahead from expanding in the Intermountain West could protect millions of acres. Managing medusahead may seem expensive per acre, but when all the acres that are protected by managing an infestation are considered the price is very reasonable. Furthermore, with escalating land prices, the cost of medusahead management to the individual livestock producer is rapidly becoming more reasonable compared to purchasing additional acreage to offset production losses from medusahead invasion. Management of medusahead is at a critical junction. Following the current path will undoubtedly result in huge economic and ecological losses with few chances for restoration. But with a serious commitment to prevention and control of new infestations, the expansion of medusahead can be greatly reduced.

Unfortunately, medusahead is not the only invasive plant species that is approaching a critical threshold. Other 
invasive plants that have the potential to occupy vast acreages and negatively impact economic and ecological yields need to be identified. Once identified, intensive prevention programs should be developed for these invasive plants. Fortunately, prevention programs can encompass several invasive plant species. Differences in dispersal mechanisms, phenology, and life cycle requirements among invasive plant species, however, suggest that some species-specific management actions may be necessary to maximize prevention effectiveness. Developing proactive programs to prevent invasive plants from invading new areas will be critical to maintaining the economic and ecological functions of rangelands.

\section{Acknowledgments}

The authors appreciate the thoughtful reviews of the manuscript by Drs Jon Bates and Ed Vasquez. The authors also thank the anonymous reviewers for their constructive reviews.

\section{References}

1. Young, J. A. 1992. Ecology and management of medusahead (Taeniatherum caput-medusae ssp. asperum [SIMK.] Melderis). Great Basin Naturalist 52:245-252.

2. Pellant, M., and C. Hall. 1994. Distribution of two exotic grasses on Intermountain rangelands: status in 1992. In: S. B. Monsen and S. G. Kitchen [comps.]. Proceedings-Ecology and Management of Annual Rangelands; 18-21 May 1992; Boise, ID, USA. Ogden, UT, USA: United States Department of Agriculture, Forest Service, Intermountain Research Station. General Technical Report INT-GTR-313. p. 109-112.

3. Turner, R. B., C. E. Poulton, and W. L. Gould. 1963. Medusahead-a threat to Oregon rangeland. Corvallis, OR, USA: Oregon State University Agricultural Experiment Station. Special Report 149. 22 p.

4. Weedmapper. 2007. http://www.weedmapper.org. Accessed 28 November 2007.

5. Hironaka, M. 1994. Medusahead: natural successor to the cheatgrass type in the northern Great Basin. In: S. B. Monsen and S. G. Kitchen. [comps.]. Proceedings-Ecology and Management of Annual Rangelands. 18-21 May 1992; Boise, ID, USA. Ogden, UT, USA: United States Department of Agriculture, Forest Service, Intermountain Research Station. General Technical Report INT-GTR-313. p. 89-91.

6. Hironaka, M. 1961. The relative rate of root development of cheatgrass and medusahead. Journal of Range Management 14:263-267.

7. George, M. R. 1992. Ecology and management of medusahead. Davis, CA, USA: University of California Range Science Report 23. p. 1-3.

8. Goebel, C. J., M. Tazl, and G. A. Harris. 1988. Secar bluebunch wheatgrass as a competitor to medusahead. Journal of Range Management 41:88-89.
9. Hironaka, M., and B. W. Sindelar. 1975. Growth characteristics of squirreltail seedlings in competition with medusahead. Journal of Range Management 28:283-285.

10. Bovey, R. W., D. Le Tourneau, and L. C. Erickson. 1961. The chemical composition of medusahead and downy brome. Weeds 9:307-311.

11. Torell, P. J., L. C. Erickson, and R. H. Haas. 1961. The medusahead problem in Idaho. Weeds 9:124-131.

12. Young, J. A., C. D. Clements, and G. Nader. 1999. Medusahead and clay: the rarity of perennial seedling establishment. Rangelands 21:19-23.

13. Kyser, G. B., J. M. DiTomaso, M. P. Doran, S. B. Orloff, R. G. Wilson, D. L. Lancaster, D. F. Lile, and M. L. Porath. 2007. Control of medusahead (Taeniatherum caputmedusae) and other grasses with Imazapic. Weed Technology 21:66-75.

14. Monaco, T. A., T. M. Osmond, and S. A. Dewey. 2005. Medusahead control with fall- and spring-applied herbicides in northern Utah foothills. Weed Technology 19:653-658.

15. Horton, W. H. 1991. Medusahead: importance, distribution, and control. In: L. F. James, J. O. Evans, M. H. Ralphs, and R. D. Child (eds.). Noxious range weeds. Boulder, CO, USA: Westview Press. p. 394-398.

16. Peterson, A. T., And D. A. Vieglais. 2001. Predicting species invasions using ecological niche modeling: new approaches from bioinformatics attack a pressing problem. BioScience 51:363-371.

17. Simberloff, D. 2003. Eradication-preventing invasions at the outset. Weed Science 51:247-253.

18. Zavaleta, E. 2000. Valuing ecosystem services lost to Tamarix invasion in the United States. In: H. A. Mooney and R. J. Hobbs (EDs.). Invasive species in a changing world. Washington, DC, USA: Island Press. p. 261-300.

19. Davies, K. W., and R. L. Sheley. 2007. A conceptual framework for preventing the spatial dispersal of invasive plants. Weed Science 55:178-185.

20. Davies, K. W. 2008. Medusahead dispersal and establishment in sagebrush steppe plant communities. Rangeland Ecology E Management 61:110-115

21. Moody, M. E., And R. N. Mack. 1998. Controlling the spread of plant invasions: the importance of nascent foci. Journal of Applied Ecology 25:1009-1021.

22. Smith, H. A., W. S. Johnson, J. S. Shonkwiler, and S. R. Swanson. 1999. The implications of variable or constant expansion rates in invasive weed infestations. Weed Science $47: 62-66$

Authors are Rangeland Scientist, United States Dept of Agriculture-Agricultural Research Service, Eastern Oregon Agricultural Research Center, 67826-A Hwy 205, Burns, OR 97720, USA, kirk.davies@oregonstate.edu (Davies); and Assistant Professor, Dept of Range Ecology and Management, Harney County Extension Office, Oregon State University, Burns, OR 97720, USA (Johnson). 